\title{
Conocimiento pedagógico del contenido para la enseñanza del tema mitosis: un estudio de casos con docentes universitarios de Argentina*
}

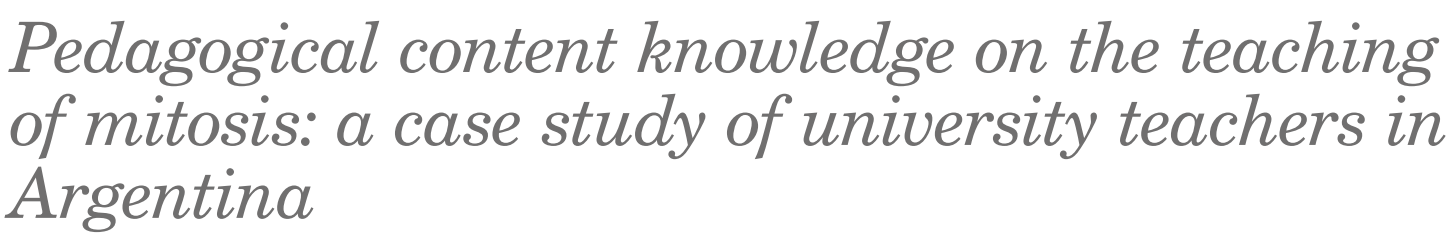

\author{
Norma V. González \\ Alejandra María Rossi \\ Universidad Nacional de La Plata, Argentina
}

Resumen: El contenido didáctico del contenido (CDC) para la enseñanza de la mitosis en la universidad es un campo poco explorado. Presentamos la caracterización el CDC de docentes universitarios sobre la mitosis empleando el cuestionario Representación del Contenido (ReCo) y los repertorios de experiencia profesional y pedagógica (Re-PyP). Las diferencias encontradas en el CDC de los docentes resultan coincidentes con las características de este constructo: es específico para un tema, un docente y un contexto. El desarrollo del CDC de los docentes universitarios en un contexto de enseñanza centrada en la transmisión de conocimientos se perfila como una estrategia posible para reorientar la enseñanza del tema mitosis hacia modalidades basadas en la construcción de andamiajes que faciliten el aprendizaje de los estudiantes.

Palabras clave: conocimiento didáctico del contenido (CDC), mitosis, universidad, representación del contenido ( $\mathrm{ReCo}$ ), repertorio de experiencia profesional y pedagógica (Re-PyP).

Abstract: The pedagogical content knowledge (PCK) for teaching mitosis at university is an unexplored field. We report the characterization of university teachers' PCK on mitosis employing the Content Representation questionnaire (CoRe) and Professional and Pedagogical experience Repertoire (PaP-eR). The differences found in the teachers' $\mathrm{CDC}$ are consistent with the characteristics of this construct: it is specific to a topic, a teacher and a context. The PCK development of university teachers in the context of teaching centered on the transmission of knowledge emerges as a possible strategy to reorient the teaching of mitosis to approaches based on the construction of scaffoldings that facilitate student learning.

KeyWords: pedagogical contentknowledge(PCK), mitosis, university,ContentRepresentation questionnaire (CoRe), Professional and Pedagogical experience Repertoire (PaP-eR).

(Fecha de recepción: marzo, 2015, y de aceptación: mayo, 2015)

DOI: 10.7203/DCES.29.4991

* Proyecto de Investigación 11/V216: "La construcción del conocimiento pedagógico del contenido (CPC) y del conocimiento del contenido tecnológico-pedagógico (CCTP) para el proceso de mitosis en profesores universitarios”, Universidad Nacional de La Plata, Argentina. Directora: Dra. Norma González. Las autoras agradecen la colaboración de los docentes participantes. 


\section{Introducción}

La mitosis es un proceso de división de las células eucariotas que se produce cuando una célula madre se divide para producir dos células hijas. Es un tema situado en la intersección de dos principios unificadores de la biología: la continuidad biológica-la reproducción de las células y los organismos- y el desarrollo -crecimiento y la reparación y regeneración de tejidos-.

El aprendizaje de este tema exige un esfuerzo considerable y los estudiantes experimentan diversas dificultades, en especial para discriminar conceptos biológicos como cromosomas, cromátidas y células diploides que requieren del pensamiento abstracto (Banet y Ayuso, 2000; Lewis y Wood-Robinson, 2000). Las dificultades de los estudiantes han sido encontradas en el nivel secundario de educación (Chattopadhyay, 2012; Jiménez Aleixandre, 2003; Riemeier y Gropengießer, 2008; Williams et al., 2012;). Varios estudios dan cuenta de la persistencia de tales dificultadesen el nivel de educación superior, en diversas carreras como Biología, Medicina y Odontología (Infante-Malaquías et $a l ., 2010$ ) y Ciencias Veterinarias (González et al., 2013a). Los propios profesores tropiezan con dificultades en el desarrollo de sus clases sobre este tema ya fueran principiantes (Yip, 1998) o experimentados (Knippels et al., 2005; Oztap et al., 2003). A su vez, su enseñanza ha sido considerada como un factor que contribuye a las dificultades de su aprendizaje (Haambokoma, 2007); los libros de texto han recibido igual consideración (Cho et al., 1985; Dikmenli, 2010).

La enseñanza y el aprendizaje de la biología celular en el nivel universitario es un campo que cuenta con facetas poco exploradas; entre ellas se encuentra el contenido didáctico del contenido (CDC) para la enseñanza del tema mitosis. En este artículo se presenta la caracterización el CDC de cuatro docentes universitarios en relación al tema mitosis mediante la aplicación del cuestionario sobre la representación del contenido ( $\mathrm{ReCo}$ ) y los repertorios de experiencia profesional y pedagógica ( $\mathrm{Re}-\mathrm{PyP}$ ).

Las preguntas que orientaron nuestra investigación fueron:

¿Cuáles son los aspectos de la mitosis que los docentes universitarios consideran centrales en la enseñanza y el aprendizaje?

¿Cómo se caracteriza el CDC de los docentes sobre la mitosis en el contexto de la universidad?

\section{Marco teórico}

El trabajo fundacional de Shulman (1986) sobre el CDC plantea que para situar el conocimiento que se desarrolla en la mente de los profesores pueden distinguirse tres tipos de conocimiento: el conocimiento del contenido temático de la materia o asignatura, el conocimiento pedagógico del contenido y el conocimiento curricular.

La descripción de Shulman del CDC estimuló la investigación sobre el conocimiento profesional de los docentes. 
Debido a que este constructo carecía de especificidad y de una base empírica, los investigadores tenían la libertad de volver a conceptualizar la idea. Así, desde la primera propuesta, la investigación del CDC dio lugar a divergencias en las definiciones, los modelos de conocimiento de los profesores y la recolección de datos (Gess-Newsome, 2013). Entre los autores que han contribuido a la elaboración de este concepto se encuentran Magnusson et al. (1999) quienes reconocen cinco componentes en el CDC, a saber: visión y propósito de la enseñanza de las ciencias, conocimiento y creencias sobre el currículo de ciencias, conocimiento y creencias acerca de la comprensión de los estudiantes sobre las ciencias, conocimiento y creencias sobre estrategias instruccionales para enseñar ciencias y conocimiento y creencias sobre evaluación en ciencias. De acuerdo a estos autores, el primer componente del CDC representa la manera general de conceptualizar la enseñanza de las ciencias. En nuestros estudios hemos utilizado como marco referencial de este componente a una caracterización de los modelos didácticos en función de la concepción curricular y epistemológica propuesta por Solís Ramírez et al. (2009). En esta tipificación se identifican cuatro modelos: tradicional o transmisivo, tecnológico, activista o espontaneísta e investigativo.

El segundo componente del CDC es el conocimiento y creencias sobre el currículo de ciencia. Consta de dos categorías: el conocimiento de los pro- fesores sobre los objetivos del currículo de la materia que están enseñando y el conocimiento de los programas curriculares específicos y de los materiales curriculares (Magnusson et al., 1999). La primera categoría comprende, además de lo mencionado, el conocimiento de los profesores sobre lo que los estudiantes han aprendido y lo que se espera que aprendan. La segunda categoría consiste en conocimientos vinculados a la existencia de programas y materiales curriculares relevantes para la enseñanza de un dominio particular de la ciencia y de tópicos específicos dentro de ese dominio. Se mencionan como ejemplos vinculados con nuestro tema de investigación los programas de laasignatura universitaria y los libros de texto empleados.

El tercero de los componentes del CDC es el conocimiento y creencias acerca de la comprensión de los estudiantes sobre las ciencias. Se incluyen en él dos categorías: los requisitos para el aprendizaje de conceptos científicos específicos y las dificultades conceptuales que los profesores identifican en su práctica (Magnusson et al., 1999). La primera categoría comprende el conocimiento y creencias sobre los saberes previos así como los diferentes estilos de aprendizaje. Respecto de la segunda categoría, estos autores mencionan que está motivado por el carácter abstracto de algunos contenidos, por las estrategias de enseñanza inadecuadas y por las concepciones alternativas que sostienen los estudiantes. 
El cuarto de los componentes del CDC es el conocimiento y creencias sobre estrategias instruccionales para enseñar ciencias. En este componente se identifican el conocimiento de las estrategias específicas para la materia y el conocimiento de las estrategias para temas específicos. Las estrategias de estas categorías difieren respecto de su alcance. Las estrategias específicas para la materia son de amplio empleo en la enseñanza de las ciencias; las estrategias para temas específicos se emplean para ciertos temas dentro de un dominio de las ciencias. Estas últimas estrategias incluyen recursos como las representaciones -ilustraciones, ejemplos, analogías, modelos- y las actividades -problemas, demostraciones, simulaciones, investigaciones y experimentos.

El quinto de los componentes del CDC es el conocimiento y creencias sobre evaluación en ciencias. Hay dos categorías de conocimiento: el conocimiento de las dimensiones del aprendizaje de las ciencias que resultan importantes evaluar y el conocimiento de los métodos de evaluación para esos aprendizajes. Las dimensiones del aprendizaje a evaluar deben estar comprendidas dentro de la alfabetización científica. El conocimiento de los métodos de evaluación debe trascenderla mera recopilación de instrumentos y procedimientos e incluir el conocimiento de las ventajas y desventajas asociadas al empleo de una modalidad de evaluación particular (Magnusson et al., 1999).

\section{Metodología}

La investigación fue un estudio de casos interpretativo y múltiple para aportar descripciones sobre los componentes del CDC y ampliar el conocimiento a partir de situaciones específicas.

El estudio tuvo lugar en una asignatura del primer año de una universidad pública en la República Argentina. Sus contenidos abarcan aspectos básicos de la biología animal y la biodiversidad. La asignatura es anual y consiste en clases teóricas y trabajos prácticos (TP). Se decidió realizar el trabajo de campo en los TP por ser el ámbito de mayor interacción entre los docentes, estudiantes y contenidos.

Cada TP se inicia con la presentación del tema por el docente. Luego los estudiantes trabajan en grupos para resolver las actividades que descargan de la página Web de la asignatura. Las observaciones de las clases se centraron la presentación del tema debido a que el trabajo grupal de los estudiantes dificultó continuar la observación sin generar incomodidades.

El tema mitosis corresponde al tercer TP. Los objetivos relacionados con la mitosis fueron: 1) diferenciar e interpretar los procesos básicos de la mitosis y meiosis y 2) analizar la importancia de la mitosis y meiosis en la vida de un organismo.

Cuatro docentes participaron voluntariamente en nuestro estudio. Su antigüedad en la docencia es mayor a veinte años; tres de ellos cuentan con forma- 
ción pedagógica. Para mayor claridad fueron identificados como $\mathrm{D}_{1}, \mathrm{D}_{2}, \mathrm{D}_{3} \mathrm{y}$ $\mathrm{D}_{4}$ en la presentación de los resultados.

La recogida de datos se realizó mediante el cuestionario de representación del contenido (ReCo) y la observación de clases para elaborar el repertorio de experiencia profesional y pedagógica (Re-PyP), ambos instrumentos propuestos por Loughran et al. (2004, 2012). El ReCo consiste en ocho preguntas:

1) ¿Qué conceptos centrales intenta que los estudiantes aprendan acerca de la mitosis?

2) ¿Por qué es importante para los estudiantes aprender el tema mitosis?

3) ¿Qué más sabe sobre este tema, pero no lo enseña porque considera que no corresponde a este nivel o a los alcances del curso?

4) ¿Cuáles son las dificultades y limitaciones conectadas con la enseñanza de este tema?

5) ¿Qué conocimientos de las ideas previas de los estudiantes acerca de la mitosis influyen en la manera en que enseña este tema?

6) ¿Qué otros factores influyen sobre la enseñanza de la mitosis?

7) ¿Qué procedimientos emplea para que los alumnos se interesen en este tema?

8) ¿Qué instrumentos utiliza para evaluar si los alumnos han comprendido el tema mitosis?

El ReCo fue presentado y discutido con los docentes luego de observar la clase. Los docentes fueron entrevista- dos individualmente a los para aclarar aspectos del ReCo y de las clases.

\section{Resultados}

ReCo. Las tres primeras preguntas abordan la orientación en la enseñanza. Las ideas centrales sobre este tema (pregunta 1) fueron:

1) La mitosis es un proceso dinámico que se divide en etapas para su estudio;

2) Los cromosomas replicados se distribuyen a las células hijas;

3) La mitosis guarda relación con la reproducción asexual.

Los docentes valoraron la importancia del tema (pregunta 2) a través de su relación con otros temas como genética $\left(D_{1}\right)$, reproducción $\left(D_{1}, D_{2}\right.$ y $D_{3}$ a nivel celular y de individuo, $\mathrm{D}_{4}$ a nivel de individuo), crecimiento y la cicatrización $\left(\mathrm{D}_{2}\right.$ y $\left.\mathrm{D}_{3}\right)$, origen de la pluricelularidad $\left(\mathrm{D}_{4}\right)$ y desarrollo embrionario $\left(D_{4}\right)$. Todos coincidieron en excluir de sus clases temas como la estructura molecular de los cromosomas (pregunta 3 ); $\mathrm{D}_{3} \mathrm{y} \mathrm{D}_{4}$ mencionaron además que prescinden de la historia de los conocimientos por la falta de tiempo.

Las preguntas 4 y 5 indagan sobre la comprensión por parte de los estudiantes.Entre las dificultades mencionadas (pregunta 4) se mencionaron la falta de conocimientos previos $\left(D_{1}, D_{2}\right.$ y $\mathrm{D}_{3}$ ) y la complejidad del vocabulario $\left(\mathrm{D}_{3}\right) . \mathrm{D}_{4}$ planteó dificultades por la heterogeneidad de los trayectos previos de los estudiantes y la ausencia de un marco teórico sobre las propiedades de 
los seres vivos que permita contextualizar a la mitosis. Como limitaciones se mencionaron la carencia de recursos motivadores que podrían mejorar la enseñanzay facilitar la comprensión del carácter dinámico y secuencial de la mitosis $\left(\mathrm{D}_{2} \mathrm{y}_{4}\right)$. Las ideas previas de los estudiantes (pregunta 5) fueron caracterizadas principalmente como conocimientos limitados y confusos.

Las preguntas 6 y 7 examinan el conocimiento de estrategias instruccionales. Las respuestas mostraron un alto grado de relación: los factores que condicionan la enseñanza (pregunta 6) fueron aludidos también como procedimiento motivador (pregunta 7). Así, $\mathrm{D}_{1}$ reconoció que el uso de maquetas puede facilitar la enseñanza; $\mathrm{D}_{2} \mathrm{y}_{3}$ mencionaron que la dificultad de los estudiantes para interpretar la mitosis con imágenes bidimensionales y estáticas se podría superar apelando a videos lo que también promovería mayor interés por el tema. $\mathrm{D}_{4}$ ante las disímiles expectativas de los estudiantes de diferentes Licenciaturas que considera condicionan su enseñanza, propuso contextualizar la mitosis en un marco biológico y apela a ejemplos y analogías para facilitar la conceptualización del tema. Finalmente, en la pregunta 8 sobre la evaluación de la comprensión de los estudiantes $\mathrm{D}_{1}, \mathrm{D}_{2} \mathrm{y} \mathrm{D}_{4}$ señalaron que la evaluación formal del tema es mediante un examen oral, al finalizar los seis primeros TP. $\mathrm{D}_{2}$ lo emplea en esa instancia como nexo para evaluar otros temas como el desarrollo embrionario y tejidos. $D_{2}, D_{3}$ y $D_{4}$ emplean preguntas orales para evaluar la comprensión durante la clase; $\mathrm{D}_{3}$ destaca el valor de las preguntas que los estudiantes hacen para identificar los aspectos que no comprendieron.

En resumen, las mayores coincidencias se encontraron en la identificación de las ideas centrales de la mitosis, la exclusión de aspectos moleculares en sus explicaciones y la relevancia del tema por su relación con otros TP. Las dificultades en la enseñanza fueron adjudicadas mayoritariamente a carencias de los estudiantes y las limitaciones a la falta de recursos motivadores. La evaluación de la comprensión del tema se realiza para dos docentes a través de preguntas orales en el transcurso de la clase y para todos ellos, de manera formal, en el examen parcial.

$R e-P y P$

Para completar la información recogida mediante el ReCo, construimos los Re-PyP de dos docentes, seleccionados sobre la base de la conexión entre sus acciones en el aula y sus concepciones evidenciadas a través del ReCo y la entrevista. Presentamos los Re-PyP de $\mathrm{D}_{3} \mathrm{y} \mathrm{D}_{4}$, a quienes identificamos de acá en adelante como Imeuda y Mauro, respectivamente.

\section{El Re-PyPde Imeuda}

Maquetas que duermen sobre las mesas de trabajo

Este Re-PyP se desarrolló a través de una narración escrita por Imeuda. 
Da cuenta de cómo unas maquetas de cromosomas llevan a una explicación que, tras atraer la atención de los estudiantes, contribuye a facilitar la comprensión de algunos aspectos ampliamente reconocidos como complejos de enseñar y de aprender.

En este tercer TP se desarrollan los temas división celular, gametogénesis y reproducción asexual y sexual. Es una clase extensa por la cantidad de contenidos a tratar a lo que se suma que es la primera vez que los estudiantes toman contacto con material preservado (esponjas, ascidias, etc.). Esta clase se relaciona con los temas del TP anterior: el flujo de la información genética y el ciclo celular. En la introducción de la clase retomo el ciclo celular con sus fases; hago un repaso como paso necesario para enmarcar los nuevos contenidos. A lo largo de los años como docente en esta cátedra, he comprobado que (a diferencia de otros temas) en el tratamiento de estos contenidos en la escuela secundaria se enfatiza el concepto de mitosis y meiosis, principalmente en las "fases". Profase, metafase, etc., son términos que los estudiantes recuerdan muy bien al igual que los principales acontecimientos que permiten caracterizar cada fase. Sin embargo, el concepto de cromátides hermanas resulta para los estudiantes indistinguible del concepto de cromosoma homólogo: no ven las diferencias entre ellos como tampoco cuándo y por qué se consideran a las cromátides hermanas. Enseñarles a interpretar el concepto de cromosoma homólogo cuesta bastante. Es por eso que, en la explicación de la división celular, me aboco a diferenciar casi exclusivamente esos conceptos: repasamos la composición química y las partes del cromosoma (centrómero, cromátides hermanas, brazo, telómeros). Hago esquemas en el pizarrón y destaco la diferencia entre los cromosomas formados por una cromátide o dos cromátides. Una pregunta que ayuda a esto es "¿cómo se verían los cromosomas en $G_{1}$, $S$ y $G_{2}$ si los pudiéramos condensar?". Voy haciendo dibujos para cada situación. Insisto en la necesidad de comprender estos conceptos para poder acceder a otros, por ejemplo les comento que es indispensable conocer todo esto para comprender de qué manera en la anafase parte del material genético va a cada célula hija y la frecuencia con que este aprendizaje no ocurre debido a la dificultad inherente al tema.

Entre las actividades de esta clase, los estudiantes utilizan maquetas de cartulina para trabajar la división celular y la gametogénesis. Hay juegos de cromosomas de diferentes formas (metacéntricos, submetacéntricos, etc.) para que modelicen células haploides, células diploides y un cariotipo. Con estas maquetas además deben realizar modelos de la división por mitosis y por meiosis. Las consignas del ejercicio son las siguientes:

Utilizando las figuras de cartulina confeccionen una célula $2 n=6$ y una célula $n=3$. Luego respondan:

¿Qué representan 2ny n?

En la célula $2 n$, ¿los cromosomas son todos iguales? ¿Cómo se disponen? 
En la célula $n$, ¿qué cromosomas están representados?

Durante muchos años vi "dormir" estas figuras de cartulina sobre las mesas de trabajo; los estudiantes no suelen ser afectos a manipularlas, consideran las tareas dificiles y piden a algún docente que las solucionen por ellos. Aquí debo hacer un alto en el relato para contar que en esta clase usamos otras maquetas similares que tienen un pequeño imán. Esas maquetas con imán fueron una idea colectiva de un grupo de docentes de otro curso en el que yo participé. La excelente respuesta de los estudiantes de aquel curso hizo que, cuando mi cargo docente me permitió tomar algunas decisiones, trajera al aula esta actividad.

Como en otras clases, los estudiantes trabajan en grupo, resolviendo las actividades de la Guía de TP y cuentan con la orientación y apoyo de los docentes. Al promediar la clase, llega el momento de "actuar". Junto a otro docente nos desplazamos hacia el fondo del aula para colocar las maquetas con imán sobre las puertas de un mueble metálico. Comenzamos con preguntas sobre el ciclo celular y el momento de duplicación del ADN. Colocamos los cromosomas en las puertas con una cromátide y para la duplicación del ADN, agregamos la cromátida hermana (Fig. I). Al llegar a la metafase ubicamos los cromosomas en la mitad de la puertas y en la anafase separamos las cromátides hermanas. Finalmente, agrupamos los cromosomas en dos células imaginarias.

\section{Figura I. \\ Las maquetas de los cromosomas empleadas por Imeuda.}

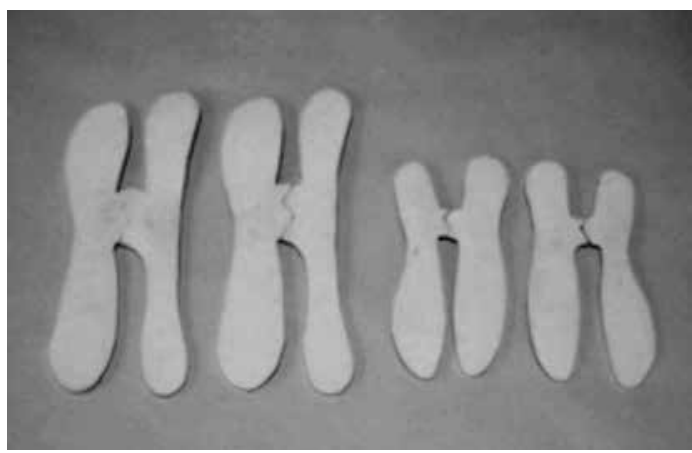

Fuente: Elaboración propia

Para la división por meiosis la propuesta es similar: utilizar las mismas maquetas. Los estudiantes disponen de un lapso de tiempo para que trabajen de la misma manera que lo hicimos. Hay cursos en los que logramos que uno o dos alumnos pasen a realizarlo y otras veces terminamos nosotros realizando la división, pero el desarrollo siempre es a través de preguntas guiadas. Gran parte del éxito de la clase recae también en los otros docentes que ayudan a generar preguntas. Dependiendo del grupo (algunos docentes son más callados o se involucran menos...) se suelen generar discusiones interesantes. La desestructuración de la clase, al trabajar sobre el mueble metálico, propicia una actitud más participativa por parte de los estudiantes.

\section{El Re-PyP de Mauro}

Un mapa conceptual en la clase expositiva 
La observación de la clase y la entrevista dieron origen al Re-PyP de Mauro; se presenta cómo desarrolla un mapa conceptual como estrategia de enseñanza.

Mauro comenzó su clase contextualizando los temas en relación a las clases anteriores y próximas clases, la herencia de los organismos y el desarrollo embrionario. Recordó a los estudiantes que "la reproducción es uno de los conceptos estructurantes de los seres vivos; es indispensable para la continuidad de los seres vivos y que se relaciona con la historia evolutiva y la diversidad celular." Luego presentó la reproducción en dos niveles de organización: celular y de organismo; estos términos encabezaban el mapa conceptual que desarrolló en un sector del pizarrón (Fig. II). Si bien hubo referencias a aspectos vinculados a la reproducción a nivel del organismo, a partir de este momento su explicación se concentró en el nivel celular.
El tratamiento de conceptos sobre el comportamiento de los cromosomas homólogos en la mitosis (y en la meiosis) fue el punto central de este tramo de la clase. Mauro realizó esquemas a medida que continuaba desarrollando el mapa conceptual. Utilizó algunas analogías; con ellas se refirió:

- A la cromatina en interfase y su condensación para la mitosis como resortes: "Imagínense que son como resortes estirados por lo tanto cuando me preparo para la mitosis..."

- A los cromosomas homólogos como "pares de zapatos": "cuando una célula diploide se divide sigo manteniendo los pares: no termino con una bota y una zapatilla; esto es incompatible con la zapatería celular".

El mapa conceptual desarrollado a medida que avanzaba por los diferentes puntos de su exposición se presenta a continuación y resume el recorrido temático de toda la clase (Fig. II).

\section{Figura II.}

\section{Mapa conceptual}

elaborado por Mauro en el transcurso de la clase.

Fuente:

Transcripción de los autores.

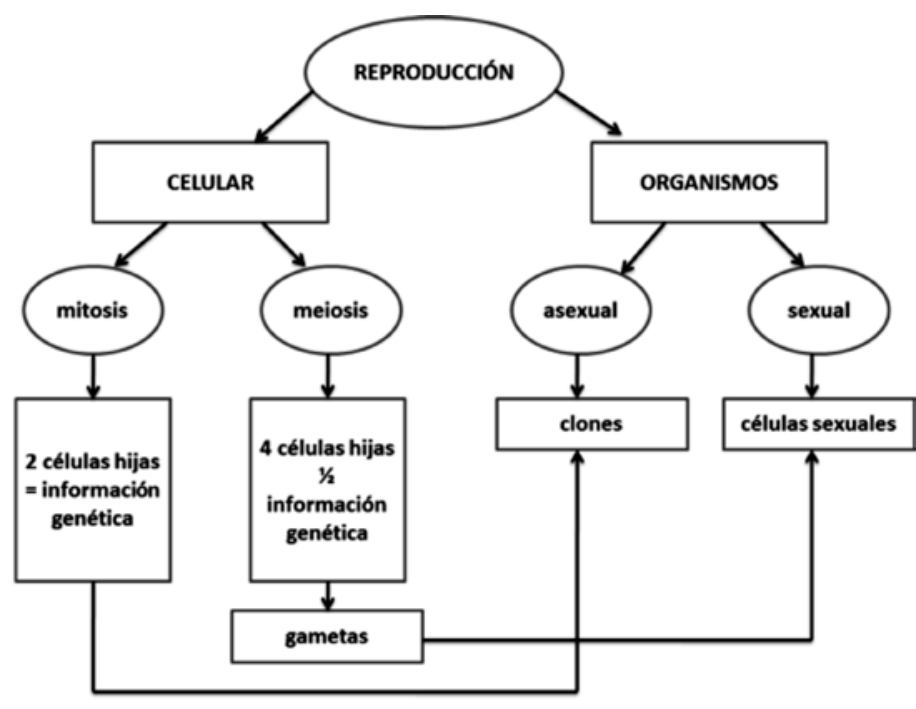


Respecto del mapa conceptual y del uso de las analogías, en el transcurso de la entrevista Mauro comentó que:

"Dos aspectos que considero imprescindibles para este y otros temas son: la contextualización del contenido en el marco de la perspectiva general (biológica y zoológica) y el planteo de interrogantes acerca del tema a comprender y aprender (problematización). Con esos propósitos y para acercarles a los estudiantes una herramienta que facilite sus aprendizajes, a lo largo de la clase construyo el mapa conceptual y utilizo ejemplos $y$ analogías que les ayuden a lograr una conceptualización adecuada."

El recorrido temático de la clase de Mauro fue diferente al de los otros docentes observados. En este sentido, nuestro docente dijo:

"En particular lo que personalmente hago es partir de los conceptos más abarcativos y estructurantes para ir profundizando conceptualmente en un recorrido cíclico de aproximación a los contenidos más específicos. Esto me permite también considerar un fenómeno que se ha venido dando en los últimos años: la diversidad de nacionalidades que se reúnen en el aula, y que se manifiesta no solo heterogeneidad de la formación de los estudiantes en cuanto a contenidos, sino también a lo referido a las diferentes lógicas de aprendizaje que responden a diferentes modelos de enseñanza."
Otro aspecto diferencial de la clase fue que, a diferencia de los otros docentes, Mauro no incluyó ejemplos de las modalidades de reproducción sexual y asexual. En el transcurso de la entrevista, fundamentó esta selección de contenidos de la siguiente manera:

"Aquí surge una ambigüedad, en el sentido de que hay contenidos que considero importantes pero que dejo fuera y otros que requieren una comprensión más profunda y que no corresponden al nivel de generalidad de la asignatura. Los primeros contenidos los refiero a las cuestiones de la historia de la ciencia y su desarrollo, así como a los aspectos tecnológicos $y$ experimentales que permitieron su descubrimiento y su estudio en la actualidad. Esto último en relación a las distintas implicancias de la mitosis en temas como diferenciación celular, regeneración, clonación $y$ reproducción vegetativa, no abordados con el detalle que tendrían en un curso de biología celular, pero si para permitir la contextualización del proceso mitótico en relación a temas concretos de actualidad científica. El segundo grupo de contenidos se refiere a cuestiones bioquímicas del funcionamiento celular y la regulación del ciclo celular."

Al finalizar la entrevista, Mauro reflexionó sobre la enseñanza del tema para este grupo particular de estudiantes:

"Algunas dificultades surgen porque los alumnos tienen expectativas 
formativas diferenciales (antropología, biología y geología), lo que hace que el interés por el tema mitosis sea muy desigual. Con el corto tiempo para desarrollarlo, sería deseable el trabajo previo sobre las propiedades de la vida y los seres vivos como sistemas biológicos haciendo énfasis el almacenamiento, conservación y transmisión de la información genética. Ahí seguramente podríamos revisar y tratar de superar dificultades conceptuales, que son variadas $y$ provienen de los recorridos formativos previos de los alumnos. Por otro lado, sería deseable contar con recursos materiales y soportes digitales que le permitan al alumno construir una visión de proceso dinámico y secuencial para la mitosis. Siento que se necesitan recursos que resulten más accesibles y atractivas a las nuevas lógicas cognitivas de nuestros alumnos (nativos digitales).”

\section{Caracterización del CDC de los docentes}

Tomando como referente los componentes del CDC que enmarcan nuestra investigación, iniciamos este apartado con la caracterización del CDC de $\mathrm{D}_{1}$ y $\mathrm{D}_{2}$ quienes que si bien como es lógico esperar, muestran matices diferenciales en los componentes de su CDC, se pueden tomar en conjunto. En relación a la manera general de conceptualizar la enseñanza de las ciencias encontramos que su accionar se encuadra mayoritariamente en el modelo didáctico tra- dicional: en una clase expositiva, con un fuerte protagonismo del profesor, presentaron información y utilizaron preguntas para indagar el conocimiento de los estudiantes. La secuencia de temas de la clase de $\mathrm{D}_{1}$ siguió el orden de las actividades que los estudiantes debían realizar. Varias veces enfatizó la importancia de aprender el vocabulario que iba anotando en el pizarrón por lo que el nivel explicativo de su discurso fue reducido y no promovió preguntas de los estudiantes. $\mathrm{D}_{2}$ concretó un recorrido que, aunque también linear, fue más variado y estimuló a los estudiantes a preguntar sobre temas relacionados, i.e. clonación.

El segundo componente del CDC es el conocimiento y creencias sobre el currículum; en este sentido, por la entrevista, $D_{1}$ y $D_{2}$ reconocen al temario de cada clase como elementos curriculares centrales y los únicos con que cuentan.

Los conocimientos y creencias acerca de la comprensión de los estudiantes sobre las ciencias corresponden al tercero de los componentes del CDC. $\mathrm{D}_{1} \mathrm{y}$ $\mathrm{D}_{2}$ adujeron que sus estudiantes necesitan poseer mejores conocimientos previos y que no los adquieren en el nivel secundario de educación. No hubo referencias a la complejidad y abstracción de la mitosis como una dificultad para los estudiantes.

El cuarto componente del CDC incluye conocimientos y creencias sobre estrategias instruccionales para enseñar ciencias. $D_{1}$ y $D_{2}$ no mencionaron estrategias concretas más allá de lo que 
habitualmente se desarrolla en el aula: exposición teórica, alguna actividad de lápiz y papel y el uso de las maquetas de cromosomas.

Por último, el quinto componente del CDC corresponde a los conocimientos y creencias sobre la evaluación en ciencias. Debido a que en las clases observadas no se realizó evaluación alguna, este componente se abordó en profundidad en las entrevistas. Los comentarios de $\mathrm{D}_{1}$ y $\mathrm{D}_{2}$ refirieron a la evaluación formal del curso que es de carácter sumativo, presencial, oral e individual.

En este punto, resulta de interés la perspectiva de Talanquer (2004) sobre el CDC; este autor sostiene que el CDC de los docentes debe ser suficiente para:

- Identificar las ideas centrales asociadas con un tema.

- Reconocer las probables dificultades conceptuales de los alumnos y el impacto en el aprendizaje.

- Identificar preguntas, problemas $o$ actividades que obliguen al estudiante a reconocer y cuestionar sus ideas previas.

- Seleccionar experimentos, problemas o proyectos que permitan que los estudiantes exploren ideas centrales en la disciplina.

- Construir explicaciones, analogías o metáforas que faciliten la comprensión de conceptos abstractos.

- Diseñar actividades de evaluación que permitan la aplicación de lo aprendido en contextos realistas y variados.

Los rasgos de los componentes del CDC que identificamos en $\mathrm{D}_{1} \mathrm{y} \mathrm{D}_{2}$, vis- tos a la luz los requerimientos de Talanquer (2004), consideramos que pueden ser reconocidos para solo los dos primeros ítems. Sobre esta base caracterizamos su CDC como incompleto y consistente en la transformación del conocimiento disciplinar orientado a la presentación simplificada de contenidos.

Proseguimos este apartado con la caracterización del CDC de los dos docentes de quienes contamos con su Re-PyP. En Imeuda encontramos la concurrencia de sus ideas sobre la enseñanza del tema vertidas en el ReCo con su accionar en clase. Su clase se desarrolló bajo un modelo didáctico tradicional; sin embargo en ella se reflejó tanto su conocimiento de la existencia de conceptos insuficientesde los estudiantes que representan barreras para la enseñanza y el aprendizaje de este contenido, como la identificación del comportamiento de los cromosomas como punto clave y de difícil comprensión. En la clase le destinó a este último aspecto un tiempo mayor respecto de otros contenidos e implementó una alternativa al uso de recursos que no resultaban efectivos. Encontramos en Imeuda la presencia parcial de los aspectos del CDC considerados por Talanquer (2004), de manera similar a lo que apreciamos para $\mathrm{D}_{1}$ y $\mathrm{D}_{2}$. Sin embargo, su alejamiento de la presentación simplificada de contenidos, el uso creativo de las maquetas junto la interacción con otros docentes y los comentarios en la entrevista nos permiten caracterizar su CDC como parcialmente desarrollado. 
Finalizamos este apartado con la caracterización del CDC de Mauro. Como reflejó el Re-PyP, su recorrido temático fue diferente en la secuenciación y en la cantidad de temas; además excluyó la descripción citológica de la mitosis abordada por $\mathrm{D}_{2} \mathrm{y}_{3}$. En un aula abarrotada de estudiantes llevó adelante una clase expositiva que enmarcó con uno de los conceptos estructurantes de la biología: la reproducción. Un concepto estructurante es un concepto cuya construcción permite adquirir nuevos conocimientos, organizar los datos de otra manera e incluso transformar conocimientos anteriores (Gagliardi, 1986). En referencia a este enfoque, Mauro comentó "El trabajo del docente está en poder pensar una propuesta adecuada para transformar un contenido disciplinar en un contenido a enseñar; los conceptos estructurantes en un curso introductorio como este permiten hacer esa transformación." En varias oportunidades él mostró su preocupación por lograr que los estudiantes consigan una mejor comprensión de la mitosis. El diseño de un mapa conceptual resultó, a su entender, una estrategia de enseñanza que promueve un ambiente de aprendizaje que espera responda a las expectativas de los estudiantes. Estas y otras facetas particulares de Mauro-que excluimos por motivos de extensión-lo muestran poseedor un CDC en el que se identifican los componentes propuestos por Magnusson et al. (1999) y Talanquer (2004). No obstante, el desenvolvimiento y la interacción de tales com- ponentes vistos en el aula, nos llevó a caracterizarsu CDC como parcialmente desarrollado: sus concepciones sobre la enseñanza se centran en la actividad de los estudiantes pero su práctica se organiza en torno al accionar y protagonismo del docente. Su comunicación con los estudiantes siguió el modelo triádico (pregunta del docente-respuesta de los estudiantes-evaluación del docente) que limitó la participación de los estudiantes a seguirlo en su exposición. De forma comparativa respecto del CDC de Imeuda, el CDC de Mauro muestra un desarrollo con una mayor interacción de los componentes relevados.

\section{Discusión}

En este estudio caracterizamos el CDC de cuatro docentes universitarios con base en la información obtenida mediante los instrumentos ReCo y Re$\mathrm{PyP}$ y una entrevista. Las categorías de análisis sobre las cuales se realizó la caracterización tuvieron como eje vertebrador a los componentes del CDC propuestos por Magnusson et al. (1999), complementados con el planteamiento de Talanquer (2004).

De acuerdo a la definición sobre el CDC y el modelo del conocimiento profesional de los docentes consensuada en un simposio internacional, el CDC se define como un atributo personal de un profesor y se considera tanto una base de conocimientos como una acción (Gess-Newsome, 2013). De particular interés para nuestra investigación resultan los amplificadores y filtros 
del modelo del conocimiento profesional que suman elementos de análisis para profundizar la caracterización de nuestros docentes. Los filtros y amplificadores son componentes que existen donde quiera que ocurra la traducción de información a través de personas o contextos. Los amplificadores pueden contribuir a potenciar la aceptación de una idea o la implementación de una acción; los filtros pueden extinguir una idea o una acción. Se han propuesto dos conjuntos de amplificadores y filtros: uno relacionado con los docentes y otro con los estudiantes. Los primeros comprenden sus creencias y orientación en la enseñanza y el contexto de enseñanza, por ejemplo los recursos didácticos $\mathrm{y}$ otras condiciones que impiden algunas formas de acción deseadas para el aula. Los amplificadores y filtros relacionados con los estudiantes incluyen factores que pueden favorecer o no sus aprendizajes, por ejemplo resistir y rechazar actividades centradas en los estudiantes o bien su compromiso con ellas (Gess-Newsome, 2013).

En nuestro análisis consideramos amplificadores los enfoques de las clases de Imeuda y Mauro. Para Imeuda las maquetas proporcionan oportunidades para que los estudiantes organicen sus conocimientos y profundicen la comprensión del tema. Para Mauro el mapa conceptual le permitió situar en un contexto biológico abarcativo diferentes temas que suelen presentarse de manera inconexa. Estos amplificadores destacan que cuando el profesor ejerce su profesión no se limita a aplicar de una manera imparcial el currículum prescripto, sino que responden teniendo en cuenta la realidad conocida desde su experiencia en el aula.

Hemos identificado como filtros relacionados con los docentes su orientación en la enseñanza, los modelos didácticos que definen su práctica y, dentro del contexto de enseñanza, el tipo de decisiones que pueden tomar. Sus concepciones sobre el aprendizaje de la mitosis son reproductivas y sus estrategias de enseñanza facilitan aprendizajes de igual carácter. El examen detallado de los datos recogidos y su contraste con las características de los modelos didácticos según Solís Ramírez et al. (2009) mostró las mayores coincidencias respecto del modelo didáctico tradicional. Este modelo ha sido considerado como un factor que influye fuertemente en la visión del profesor acerca los otros componentes del CDC, i.e. conocimientos sobre la comprensión de sus estudiantes y la evaluación en ciencias (Friedrichsen et al., 2009). Otro filtro identificado, derivado del contexto institucional y sus condicionantes, es el rango de decisiones que pueden tomar estos docentes: el uso de recursos como videos y animaciones, por prescripción de sus superiores, está restringido a las clases teóricas.

Respecto de los filtros vinculados a los estudiantes, durante las observaciones encontramos que los estudiantes, si bien relegados al papel de oyentes en la primera fase del TP, no generaron más que el murmullo de fondo que suele ser habitual para clases tan 
numerosas, acompañado por el uso de teléfonos móviles. Esta situación deja interrogantes en referencia al atractivo de las clasesy al tiempo destinado a la presentación de contenidos que redunda en escasos aprendizajes.

Deseamos cerrar esta discusión con una reflexión acerca de la asunción del CDC como un continuo ya que ella conlleva una segunda hipótesis: el CDC puede fortalecerse (Borowski et al., 2012). En investigaciones con profesores principiantes se ha señalado que la interacción de los componentes del CDC es limitada (Friedrichsen et al., 2009; González y Rossi, 2014) y que el CDC de un profesor evoluciona a lo largo de su práctica profesional (Olander y Olander, 2013). Nuestra caracterización del CDC de los docentes participantes como incompleto $\left(\mathrm{D}_{1} \mathrm{y}_{2}\right)$ y parcialmente desarrollado $\left(\mathrm{D}_{3} \mathrm{y}_{4}\right)$ señala la necesidad de prestar especial atención a la construcción de CDC como forma de mejorar el conocimiento profesional (Loughran et al., 2012). Habida cuenta del reconocido papel de la reflexión en el desarrollo del CDC de profesores de ciencias (Popovic y Antink, 2010) destacamos la pertinencia de incluir instancias de formación profesional y actualización en servicio para mejorar el desarrollo profesional de los docentes universitarios.

\section{Conclusiones}

- Los Re-PyP presentados son autónomos por cuanto ilustran cómo el CDC se hace evidente al revelar cada uno de ellos aspectos concretos del ReCo en el accionar en el aula (Imeuda) y en la reflexión en la acción y sobre la acción (Mauro).

- Las diferencias encontradas en el CDC de los docentes resultan coincidentes con las características de este constructo: el CDC es específico para un tema, un docente y un contexto.

- El desarrollo del CDC de los docentes en este contexto universitario con una enseñanza tradicional centrada en la transmisión de conocimientos se perfila como una estrategia posible para re-orientar la enseñanza del tema mitosis hacia modalidades basadas en la construcción de andamiajes que faciliten el aprendizaje de los estudiantes.

- Ante un constructo como el CDC resultan reducidos los estudios sobre este aspecto del conocimiento profesional de los docentes universitarios, situación que es particularmente notoria para la mitosis, el tema que nos ocupa. Presentamos en este trabajo un tramo de investigación mayor que tiene como uno de sus objetivos específicos documentar el CDC de los profesores universitarios para el tópico mitosis con la convicción de la pertinencia de llevar adelante propuestas que brinden información sobre la transformación que realizan los docentes universitarios del saber disciplinar en estructuras comprensibles para sus estudiantes. 


\section{Referencias bibliográficas}

BANET, E. y AYUSO G.E. (2000). Teaching Genetics at Secondary School: a strategy for teaching about the localization of inheritance information.ScienceEducation,84(3), 313-351. DOI: 10.1002/(SICI)1098$237 \mathrm{X}(200005) 84: 3<313$ :AID SCE2>3.0.CO;2-N.

BARRASS, R. (1984). Some misconceptions and misunderstandings perpetuated by teachers and textbooks of biology. Journal of Biological Education, 18 (3), 201-206. DOI:10.1080/ 00219266.1984 .9654636$.

BOROWSKI, A., CARLSON, J., FISCHER, H.A., HENZE, I., GESSNEWSOME, J., KIRSCHNER, S. yVAN DRIEL, J. (2012). Different models and methods to measure teachers' pedagogical content knowledge. En C. BRUGUIÈRE, A. TIBERGHIEN y P. CLÉMENT (eds.), E-Book Proceedings of the ESERA 2011 Conference: Science learning and Citizenship. (pp.37-40). Recuperado de http://www.esera. org/media/ebook/strand13/ebookesera2011_BOROWSKI-13.pdf [1 de marzo de 2012].

CHATTOPADHYAY, A. (2012). Understanding of Mitosis and Meiosis in Higher Secondary Students of Northeast India and the Implications for Genetics Education. Education, 2(3), 41-47. DOI: 10.5923/j. edu.20120203.04.
CHO, H.; KAHLE, J.B. y NORDLAND, F.H. (1985). An investigation of high school biology textbooks as sources of misconceptions and difficulties in genetics and some suggestions for teaching genetics. Science Education, 69 (5), 707-719. DOI:10.1002/ sce.3730690512.

DIKMENLI, M. (2010). Misconceptions of cell division held by studentteachers in biology: a drawing analysis. Scientific Research and Essay, 5 (2), 235-247.

FRIEDRICHSEN, P.J.; ABELL, S.K.; PAREJA, E.M.; BROWN, P.L.; LANKFORD, D.M. y VOLKMANN, M.J. (2009). Does Teaching Experience Matter? Examining biology teachers' prior knowledge for teaching in an alternative certification program. Journal of Research in Science Teaching, 46 (4), 357-383. DOI:10.1002/tea.20283.

GAGLIARDI, R. (1986). Los conceptos estructurales en el aprendizaje por investigación. Enseñanza de las Ciencias, 4 (1), 30-35.

GESS-NEWSOME, J. (2013). The PCK summit consensus model and definition of pedagogical content knowledge.ESERA 2013 (X Congreso European Science Education Research Association). Recuperado de http://www.esera2013.org.cy/ programme/detailed-programme/ parallel-sessions/?session=15 [12 de octubre de 2013].

GONZÁLEZ, N.V.; DIESSLER, M.; WOUDWYK, M. y ROSSI, A.M. 
(2013a). Enseñanza del tema mitosis en la universidad: el conocimiento pedagógico del contenido de dos docentes de biología celular. II Simposio de Enseñanza de Biología de la Facultad de Humanidades y Ciencias de la Educación. Universidad Nacional de La Plata.

GONZÁLEZ, N.V.; DIESSLER, M. y ROSSI, A.M. (2013b). A case study exploring a university teacher's pedagogical content knowledge on mitosis. Proceedings 10 th Conference of the European Science Education Research Association (ESERA). Recuperado de http:// www.esera2013.org.cy/programme/ detailed-programme/parallelsessions/?session=13 [3 de noviembre de 2014].

GONZÁLEZ, N.V. y ROSSI, A.M. (2014).

La enseñanza de la meiosis en el nivel secundario: el conocimiento didáctico del contenido de profesores expertos y principiantes. En: A. GARRITZ, S. DAZZA y M.G. LORENZO (eds.), Conocimiento Didáctico del Contenido. Una perspective Iberoamericana, (403-453). Saarbrücken: Editorial Académica Española.

HAAMBOKOMA, C. (2007). Nature and Causes of Learning Difficulties in Genetics at High School Level in Zambia. Journal of International Development and Cooperation, 13(1), 1-9.

INFANTE-MALACHIAS,M.E.;MELLO PADILHA, I.Q.; WELLER, M. y SANTOS, S. (2010). Comprehension of basic genetics concepts by Brazilian undergraduate students. Revista Electrónica de Enseñanza de las Ciencias, 9, 657-668.

JIMÉNEZ ALEIXANDRE, MP. (2003). La enseñanza y el aprendizaje de la biología. En: M.P. JIMÉNEZ ALEIXANDRE Enseñar ciencias (119-146). Barcelona: Graó.

KNIPPELS, M.C.; AREND, J.W. y KERST, T.B. (2005). Design criteria for learning and teaching genetics. Journal of Biological Education, 39, 108-112. DOI:10.1080/00219266.200 5.9655976 .

LEWIS, J. y WOOD-ROBINSON, C. (2000). Genes, chromosomes, cell division and inheritance-do students see any relationship? International Journal of Science Education, 22(2), 177-195. DOI: 10.1080/095006900289949.

LOUGHRAN, J.J.; MULHALL, P. y BERRY, A. (2012).Understanding and Developing Science Teachers' Pedagogical Content Knowledge. $2^{\text {nd }}$ ed. Rotterdam, The Netherlands: Sense Publishers.

LOUGHRAN, J.J.; MULHALL, P. y BERRY, A. (2004). In Search of PCK in Science: Developing Ways of Articulating and Documenting Professional Practice. Journal of Research in Science Teaching, 41(4), 370-391.

MAGNUSSON, S.; KRAJCIK, J. y BORKO, H. (1999). Nature, sources, and development of pedagogical 
content knowledge for science teaching.En J. GESS-NEWSOME y N G. LEDERMAN (eds.), Examining pedagogical content knowledge (95132). Dordrecht: Kluwer Academic Publishers.

OLANDER, C. y OLANDER, M.H. (2013). Professional Development Through the Use of Learning study: contributions to pedagogical content knowledge in biology. Procedia Social and Behavioral Sciences, 89, 205-212. DOI:10.1016/j.sbspro.2013.08.835.

OZTAP, H.; OZAY, E. y OZTAP, F. (2003). Teaching cell division to secondary school students: an investigation of difficulties experienced by Turkish teachers. Journal of Biological Education, 38, 13-16. DOI: 10.1080/00219266.2003.9655890.

POPOVIC, G. y ANTINK, A. (2010). Exploring the relationship between subject matter knowledge and reflective practice among preservice science and mathematics teachers. Recuperado de http://files.ecetera.si/ IOSTE/419.pdf [15 de diciembre de 2012].

RIEMEIER, T. y GROPENGIEßER, H. (2008). On the Roots of Difficulties in Learning about Cell Division: Process-based analysis of students' conceptual development in teaching experiments. International Journal of Science Education, 30(7), 923-939. DOI: 10.1080/09500690701294716.

SHULMAN, L.S. (1986). Those who understand: Knowledge growth in teaching. Educational Researcher, 15, 4-14.

SOLÍS RAMÍREZ, E., RIVERO GARCÍA, A., y MARTÍN DEL POZO, R. (2009). La presencia y el papel del activismo en las concepciones del Profesorado de Ciencias de Secundaria en Formación Inicial. Investigación en la Escuela, 67, 37-49.

TALANQUER, V. (2004). Formación Docente: ¿Qué conocimiento distingue a los buenos maestros de química? Educación Química, 15 (1), 52-58.

WILLIAMS, M.; DEBARGER, A.H.; MONTGOMERY, B.L.; ZHOU,X. y TATE, E. (2012). Exploring Middle School Students' conceptions of the relationship between genetic inheritance and cell division. Science Education, 96 (1), 78-103.

YIP, D. (1998). Identification of misconceptions in novice biology teachers and remedial strategies for improving biology learning. International Journal of Science Education, 20(4), 461-477. 